to 42.9), $\mathrm{p}=0.001)$, respectively. No enlargement of mean maximal target lesion diameter $(\mathrm{mm})$ in group A (before vs. after; 61.37?20.1 vs 64.25?22.0 ( $\mathrm{P}=0.099)$, but significant increase in group B (50.1?21.1 vs. 55.4?18 ( $\mathrm{p}=0.017)$, respectively).No significant difference of 6 -month survival rate.

Conclusions EUS-RFA plus concurrent CMT significantly reduce morphine dosage requirement than CMT in UPC. RFA additionally stabilized the tumor maximal target diameter whereas CMT failed.

\section{IDDF2019-ABS-0172 GUT MICROBIOTA SHIFT AND LOW FIBRE INTAKE IN POST GESTATIONAL DIABETES WOMEN}

\begin{abstract}
'Zubaidah Hasain*, ${ }^{2}$ Nor Azmi Kamaruddin, ${ }^{3}$ Nor Azlin Mohamed Ismail, ${ }^{4}$ Tong Seng Fah, ${ }^{5}$ Nurul Huda Razalli, ${ }^{1}$ Norfilza Mohd Mokhtar, ${ }^{2}$ Raja Affendi Raja Ali. ${ }^{1}$ Department of Physiology, Faculty of Medicine, Universiti Kebangsaan Malaysia, Kuala Lumpur, Malaysia; ${ }^{2}$ Department of Medicine, Faculty of Medicine, Universiti Kebangsaan Malaysia Medical Centre, Kuala Lumpur, Malaysia; ${ }^{3}$ Department of Obstetrics and Gynaecology, Faculty of Medicine, Universiti Kebangsaan Malaysia Medical Centre, Kuala Lumpur, Malaysia; ${ }^{4}$ Department of Family Medicine, Faculty of Medicine, Universiti Kebangsaan Malaysia Medical Centre, Kuala Lumpur, Malaysia; ${ }^{5}$ Dietetic Programme, Faculty of Health Sciences, Universiti Kebangsaan Malaysia, Kuala Lumpur, Malaysia
\end{abstract}

\subsection{6/gutjnl-2019-IDDFabstracts. 180}

Background Women with previous history of gestational diabetes mellitus (GDM) have unhealthy dietary patterns and profound gut microbiota shift. They have seven-fold higher risk to develop type 2 diabetes mellitus (T2DM) in the future. We hypothesised that probiotics intervention will modulate gut microbiota and reverse glucose intolerance (GI) in post GDM women. The aim of the study was to investigate the dietary patterns and gut microbiota composition in post GDM women. Secondly, we aimed to determine the effects of probiotics on the baseline anthropometric and biochemical markers of post GDM women.

Methods Baseline clinical characteristics including glucose tolerance assessment, anthropometric measurement and a 3-day dietary record of 45 post GDM women were obtained. Post GDM women were grouped based on glucose tolerance [normal glucose tolerance (NGT) and glucose intolerance (GI)]. 36 participants were assigned for 12 weeks of either probiotics or placebo intervention. Anthropometric and biochemical markers of pre and post-treatment were evaluated. Faecal samples were sent for $16 \mathrm{~S}$ sequencing pre and posttreatment.

Results $42.2 \%$ of 45 post GDM women have postpartum GI and significantly obese as compared with the NGT group $(\mathrm{p}<0.01)$. Mean daily fiber intakes of post GDM women was significantly suboptimal according to the Malaysian dietary recommendation $(\mathrm{p}<0.001)$. Pre-treatment, total cholesterol and high-sensitivity C-reactive protein (hsCRP) of 36 participants were above the recommendation value (4.95 ? 1.01 $\mathrm{mmol} / \mathrm{L}, 5.54$ ? $5.29 \mathrm{mg} / \mathrm{L})$. Post-treatment, change in body mass index (BMI), waist-hip ratio (WHR), HbA1c and hsCRP of ten participants differed significantly between probiotics and placebo group $(\mathrm{p}<0.05)$. At the phylum level, the gut composition of 12 post GDM women was enriched with an abundance of Bacteroidetes and a relatively small proportion of Firmicutes, Verrucomicrobia, and Proteobacteria. Based on the genus level, relative abundance of Prevotella_9 genus in post GDM women with GI was $20.1 \%$ compared to only $2.1 \%$ in NGT group.

Conclusions Post GDM women with glucose intolerance were obese, consumed suboptimal fibre and have gut microbiota shift similar to the T2DM adult. Roles of probiotics in post GDM women needs further validation.

\section{IDDF2019-ABS-0173 SYSTEMATIC REVIEW OF CALCINEURIN INHIBITORS (CNI) AND VEDOLIZUMAB (VDZ) COMBINATION THERAPY IN ACUTE SEVERE ULCERATIVE COLITIS (ASUC)}

Huiyu Lin*, Wee Chian Lim. Tan Tock Seng Hospital, Singapore

\subsection{6/gutjnl-2019-IDDFabstracts. 181}

Background Patients with acute severe ulcerative colitis (ASUC) may be refractory to treatment with steroids and antitumour necrosis factor agents (anti-TNF). Ciclosporin inhibitors (CNI) have been used effectively as a fast-acting bridge to slower-onset immunomodulators in thiopurine-naive patients; concerns over toxicity limit prolonged use as maintenance. Patients who are azathioprine-exposed or anti-TNF-refractory have limited medical treatment options, often resulting in colectomy. Combination of CNI as induction and slower-acting but potentially safer vedolizumab (VDZ) has recently been used in patients with severe inflammatory bowel disease (IBD). We aim to review the utility in ASUC.

Methods A systematic bibliographic review was conducted on PubMed using the keywords "vedolizumab", "calcineurin inhibitors", "inflammatory bowel disease", "severe ulcerative colitis" within the period 2013 to October 2018.

Results There were 2 prospective observational studies (1-2) $[\mathrm{N}=30]$ and 1 retrospective study ${ }^{(3)}[\mathrm{N}=39]$. Patients were refractory to conventional treatment with steroids and/or antiTNF therapy. CNI (ciclosporin or tacrolimus) was used for induction of remission in majority of cases, or as rescue agent in those failing induction with Vedolizumab [subgroup of 1 study, $\mathrm{N}=7$ ].

In 2 studies, IV cyclosporine or Tacrolimus was started; a week later, CNI-responsive patients were given VDZ induction/maintenance and CNIs were stopped after 8-12 weeks per protocol. In another study, VDZ was initiated on average 30days after CNI, with average combination CNI+VDZ of 64 days

Combination CNI+VDZ showed good short-term efficacy. At 1 year, there was a respectable colectomy-free rate of $75 \%$, comparable to other studies with infliximab/ciclosporin combined with azathioprine. In those receiving steroids at baseline, Steroid-free remission was achieved in 18/36 $=50 \%$ at week 14. Serious adverse events $(N=7)$ were attributed to CNIs; there were no deaths.

Conclusions Preliminary studies of combination CNI and VDZ in patients with ASUC appear promising. Further prospective trials are needed for the confirmation of the utility and efficacy of this treatment strategy in the management of ASUC 